\title{
Impactos en el proceso productivo de las pasifloras y uso eficiente del agua de riego
}

\section{Impacts on the productive process of passion flower and efficient use of irrigation water}

\author{
Armando Torrente Trujillo ${ }^{1}$ \\ armando.torrente@gmail.com. \\ Edinson Mujica Rodríguez ${ }^{2}$ \\ edinsonagricola@gmail.com. \\ ${ }^{1}$ Universidad Surcolombiana \\ ${ }^{2}$ Grupo de Investigación Hidroingeniería y Desarrollo Agropecuario - GHIDA, Universidad \\ Surcolombiana
}

Artículo recibido en octubre de 2016, arbitrado enero de 2017 y publicado en mayo de 2017

RESUMEN

Para contribuir con el desarrollo tecnológico y productivo del cultivo de pasifloras y proponer un modelo de adaptación al cambio climático y uso eficiente del recurso hídrico, se evaluó la eficiencia técnica, socioeconómica y de impactos ambientales de los sistemas de riego en la actividad productiva de los cultivos de maracuyá (passiflora edullis) y granadilla (passifloras ligularis Juss) en el departamento del Huila. Para la evaluación de los sistemas de riego en pasifloras se aplicó la metodología de Cristiansen 1942, Keller y Karmeli 1975, Gurovich 1993 y Arboleda 1994. Se propusieron medidas para evitar o mitigar los impactos ambientales, resultando los métodos de riego por goteo, microaspersión y surcos controlado con la mayor eficiencia y acogida para el suministro de agua a las pasifloras. Los impactos positivos anotados con mayor frecuencia son el aumento en la producción, el suministro de agua al cultivo en forma oportuna y adecuada, y la oportunidad para la aplicación de fertilizantes; los impactos negativos de mayor incidencia son la proliferación de malezas, las plagas y enfermedades, y las afecciones al suelo especialmente por erosión. Se recomiendan acciones para el manejo fitosanitario, la capacitación sobre riego y el mantenimiento preventivo de los sistemas de riego. Se sugiere disponer de las unidades de filtrado en el sistema de riego, el debido control de la escorrentía y la estimación de la lámina de riego adecuada para las necesidades del cultivo.

Palabras clave: Métodos de riego; Pasifloras; Impactos ambientales; coeficientes de riego
ABSTRACT

To contribute to the technological and productive development cultivation of Passiflora and propose a model of adaptation to climate change and efficient use of water resources, we assessed the technical efficiency and environmental impacts of irrigation used in crop production activities passion fruit "Passiflora edullis" and passion "passifloras ligularis Juss" in producing municipalities of Huila. For technical and environmental evaluation methodology was applied Cristiansen 1942, Keller y Karmeli 1975, Gurovich 1993 and Arboleda 1994. Proposed measures to avoid or mitigate environmental impacts, resulting in the drip irrigation method, micro-sprinkler and controlled as efficiently and welcome to supply water to the Passiflora. The positive impacts are the most frequently noted the increase in production, supply of water to the crop in a timely and appropriate, and the opportunity for the application of fertilizers, the negative impacts of increased incidence are the proliferation of weeds, pests and diseases and conditions on the ground especially by erosion. Actions are recommended for plant health management, training, irrigation and preventive maintenance of irrigation systems. It is suggested to have the filtering units in the irrigation system, proper control of runoff and the estimated water depth suitable for crop requirements.

Key words: Irrigation methods, Passifloras, Environmental, Irrigation efficiency 


\section{INTRODUCCIÓN}

En Colombia, la granadilla y el maracuyá son dos productos agrícolas representativos de las pasifloras, alimentos de mayor producción en el mercado que significa cerca de 9,5 millones de dólares de ingresos al año. El Huila es el mayor productor de passifloras de Colombia, ya que cuenta con cerca de 2.500 hectáreas cultivadas y una representatividad del $28 \%$ de la producción, este departamento se ha proyectado en los últimos años como uno de los más pujantes en producción y comercialización de frutales; la estructura productiva se califica rezagada en lo tecnológico, lo cual implica un serio riesgo para mantenerse en los mercados (MADR et al, 2006).

En la actualidad, las áreas productivas de pasifloras en el departamento del Huila presentan baja sostenibilidad y están expuestas a los múltiples riesgos técnicos socio-económicos y ambientales. Uno de los factores que inciden de manera importante sobre la sostenibilidad de las pasifloras es la dotación y el control del suministro de agua de riego a las plantaciones. Las áreas bajo producción de granadilla y maracuyá presentan en su mayoría riego tradicional o instalaciones rudimentarias que no garantizan la eficiencia y la uniformidad en la aplicación de agua a los cultivos. Por lo anterior, la agenda prospectiva de la cadena de pasifloras en el Huila determinó como acción prioritaria, la estimación de las necesidades hídricas en los cultivos de granadilla y maracuyá, como la evaluación de los sistemas de riego para conocer el estado de funcionamiento $y$ promover el uso eficiente del agua.

La evaluación de un sistema de riego conlleva una serie de mediciones de campo con el propósito de diagnosticar su funcionamiento y las posibles causas de problemas asociados al riego en el área de cultivo. Se hace necesario entonces, conocer las características básicas de los productores de pasifloras y cuáles son las afecciones durante el ciclo productivo de los cultivos. Según las experiencias de Llontop (1999) en el Perú, desde el punto de vista social, el cultivo de granadilla se ha convertido en el principal componente del mejoramiento del nivel de vida.

Las actividades agrícolas generan importantes impactos ambientales a los ecosistemas, aunque algunas no se perciban con claridad, los sistemas de riego no son la excepción y pueden ocasionar afecciones a los recursos naturales como es el suelo, las fuentes de agua, la atmósfera y al cultivo con incidencia en la rentabilidad económica de las familias y la producción departamental.

\section{MATERIALES Y MÉTODOS}

Para la investigación se instalaron parcelas experimentales de maracuyá "pasiflora edullis" y granadilla "passiflora ligularis Juss" en el suroccidente del departamento del Huila localizadas en la vereda Fátima (1200 msnm) del municipio de la Plata y en la vereda Betania (1808 msnm) del municipio de la Argentina, con la siguiente dotación: tanques para el almacenamiento de agua, estaciones climatológicas automatizadas, lisímetros volumétricos, sectores de riego (goteo, microaspersión, microtubo, surcos y tradicional), y tensiómetros instalados a 15 y $30 \mathrm{~cm}$ de profundidad por sector. En esta parcela de la Argentina se implementó el método de riego por microtubo en lugar del riego por surcos, debido a las limitaciones topográficas, pendiente superior al $30 \%$ y a las características inestables del suelo (Andisol).

La evaluación técnica, socio-económica y ambiental de los sistemas de riego siguió la metodología de Cristiansen 1942, Keller y Karmeli 1975, Gurovich 1993 y Arboleda 1994 con aplicación de encuestas a productores del sector sobre la operación de los sistemas de riego, los impactos ambientales y la calificación ambiental bajo tres escenarios posibles relacionados con las 


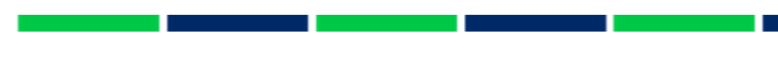

principales opciones de riego uniformidad de distribución (UD) para caudal (microaspersión, goteo y surcos).

Evaluación técnica del riego. Los problemas técnicos en los sistemas de riego afectan la eficiencia y generan impactos ambientales que inciden sobre la productividad y la calidad de la fruta, teniendo efectos sobre los aspectos socio-económicos de los productores de pasifloras. A continuación se describe la metodología para la evaluación del riego:

Microaspersión. En el sector de microaspersión se instalaron filtros de discos, tuberías laterales PE- $16 \mathrm{~mm}$ de diámetro y microaspersores tipo FranJet de 40 lph con diámetro húmedo de $5 \mathrm{~m}$. En la evaluación del riego los parámetros tenidos en cuenta son: el coeficiente de uniformidad (CU) y la

y presión.

Se midió la descarga a distintas presiones con el propósito de estimar las diferencias entre los datos medidos y los especificados por el fabricante. Para el coeficiente de uniformidad (CU) de los caudales y las presiones del sistema, se siguió la metodología de Christiansen (1942), la cual consistió en trazar en cuadrícula un área total de $2 \mathrm{~m} \times 2 \mathrm{~m}$ alrededor del microaspersor sobre el lateral crítico seleccionado; en los vértices de la cuadrícula se colocaron los recipientes pluviométricos de igual área colectora ( $7 \mathrm{~cm}$ de diámetro), en los que se midió el agua emitida por el microaspersor durante una hora (figura 1).
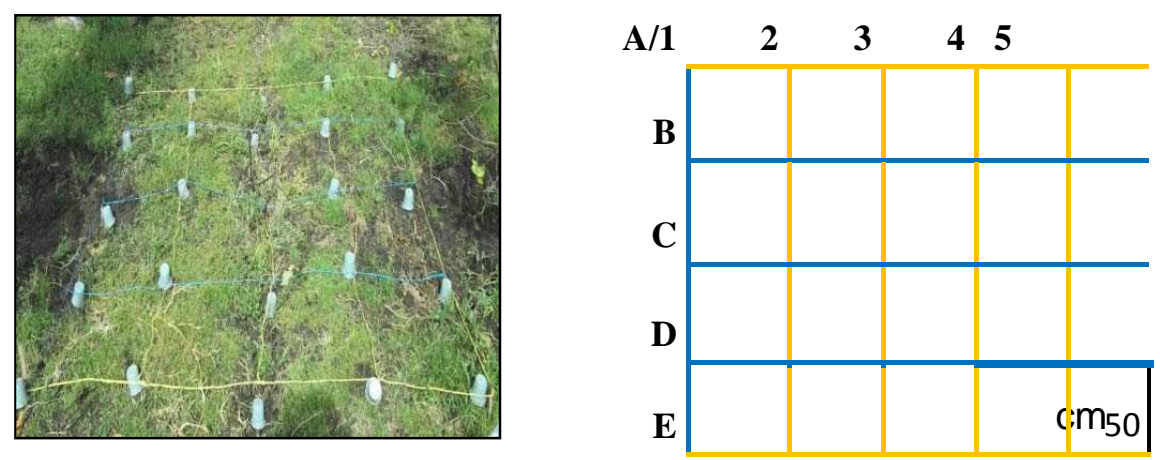

Figura 1. Disposición en cuadricula de los recipientes pluviométricos

El coeficiente de uniformidad propuesto por Christiansen, 1942 es:

$$
\mathrm{CU}=100\left[1-\frac{\sum\left|\mathrm{x}_{\mathrm{i}}-\overline{\mathrm{x}}\right|}{\overline{\mathrm{x} n}}\right]
$$

Donde CU es el coeficiente de uniformidad de Christiansen, en \%; $|x i-x|$ es la desviación absoluta de las observaciones de precipitación individuales respecto a la media, en $\mathrm{mm}$; $\mathrm{xi}$ son las observaciones individuales de lámina de agua captada en cada punto de una malla regular de colectores de precipitación del sistema, en $\mathrm{mm}$; $\mathrm{x}$ es la precipitación media, en $\mathrm{mm}$; $\mathrm{n}$ es el número de observaciones.
El coeficiente de uniformidad en la distribución que se utilizó para la evaluación de los sistemas de riego fue el propuesto por Keller y Karmeli (1975):

Donde UD es el coeficiente de uniformidad en la distribución en \%; qmin es caudal mínimo del $25 \%$ del total de los distribuidores en lph; q es el caudal medio de todos los distribuidores en lph. Se seleccionó 
el lateral crítico (mayor elevación) que contiene nueve (9) microaspersores. Se midieron los caudales y las presiones en las siguientes posiciones: la primera salida, a un tercio (1/3), a dos tercios $(2 / 3)$ y la última salida. La presión se midió con manómetro, mientras que la descarga de los emisores por el método volumétrico (figura 2).

$\mathrm{UD}=$

$\left[\frac{\mathrm{q}_{\min }}{\overline{\bar{q}}}\right]$

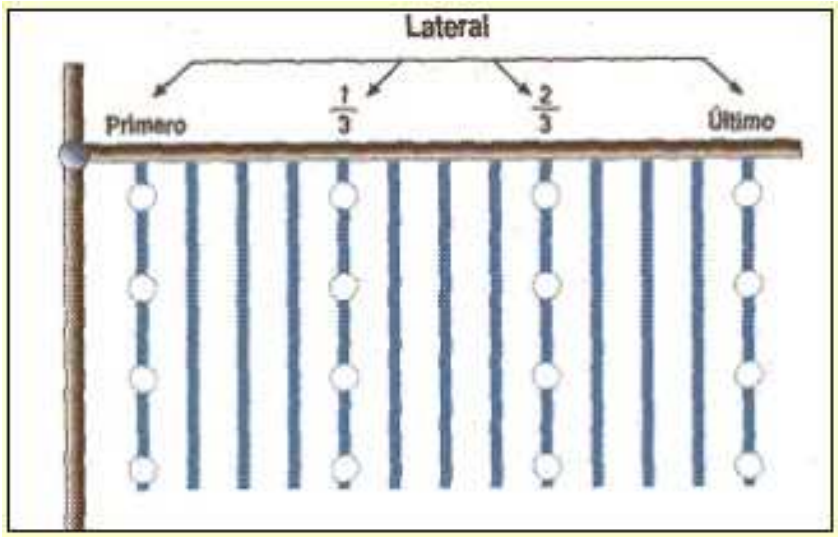

Figura 2. Puntos de evaluación en riego por goteo en la evaluación de campo

Goteo. En el sistema de riego por goteo se evaluó la descarga (q) y la presión (p) para calcular el coeficiente de uniformidad de caudales (CUc) y el coeficiente de uniformidad de presiones (CUp) en 16 emisores distribuidos uniformemente en la unidad de riego. Los goteros son de flujo turbulento de 4 lph Irriplast1. Se seleccionaron 4 laterales sobre el múltiple así: inicial, 1/3, 2/3 y final.
Seleccionados los laterales se escogieron 4 goteros por lateral, los goteros están espaciados cada $30 \mathrm{~cm}$ e insertados en tubería $\mathrm{PE}$ - diámetro $16 \mathrm{~mm}$ dispuesta de manera paralela a la hilera de plantas. Para medir la presión se instalaron derivaciones próximas a los goteros con conectores, micromanguera y obturador (ver figura 3).
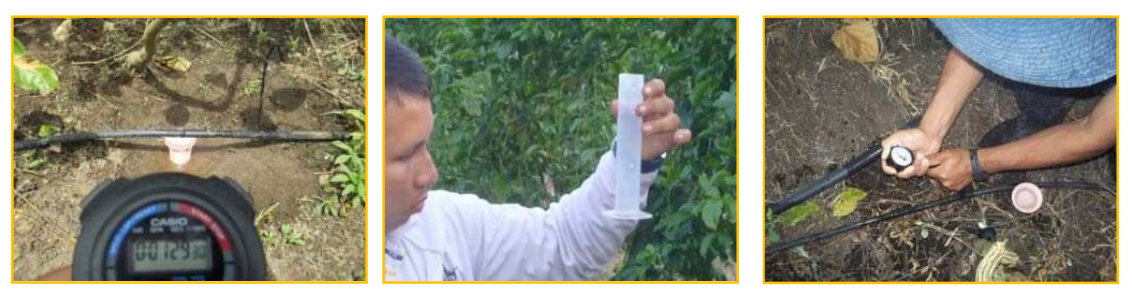

Figura 3. Evaluación técnica del riego por goteo. Medición del tiempo, volumen y presión 
Surcos. En la evaluación del sistema de riego por surcos se siguió la metodología propuesta por Gurovich (1993), que consiste básicamente en encontrar el caudal óptimo no erosivo. Se efectuaron las pruebas correspondientes a tiempo de avance e infiltración para determinar el tiempo de riego, el volumen de agua y la eficiencia de aplicación. Las pruebas de infiltración y de avance se practicaron en un surco representativo.

Microtubo. Se compone de los laterales (PE de $16 \mathrm{~mm}$ ) sobre los cuales se inserta un microtubo de $5 \mathrm{~mm}$ que descarga un flujo de tipo laminar sobre el plateo de la planta. En la parcela experimental de la Argentina se evaluó el método de riego por microtubo mediante el aforo volumétrico a lo largo de los laterales.

Tradicional. Está constituido por un hidrante para captación y conexión de la fuente de agua y manguera con suficiente longitud para colocar durante un tiempo limitado a cada planta sobre el área de cultivo. Tanto el tiempo como la descarga son muy variables para cada planta y sobre cada sector de riego.

Calificación del riego. Para la calificación del coeficiente de uniformidad y la uniformidad de distribución se aplicó la clasificación de Christiansen 1942 (ver tabla $1)$.

Tabla 1. Calificación del Coeficiente de Uniformidad y la Uniformidad de Distribución de los métodos de riego evaluados

\begin{tabular}{|l|l|}
\hline CU, UD (\%) & Calificación \\
\hline$>95$ & Excelente \\
\hline $85-95$ & Buena \\
\hline $80-85$ & Aceptable \\
\hline $70-80$ & Pobre \\
\hline$<70$ & Inaceptable \\
\hline
\end{tabular}

La calificación ambiental y ponderación de los impactos ambientales siguió la metodología de Arboleda (1994) y se proyectó para tres escenarios: microaspersión (E1), goteo (E2) y surcos (E3). Para la representatividad del impacto se estudian cinco factores: clase, presencia, duración, evolución y magnitud. La calificación ambiental en los escenarios se obtiene según los rangos que determina la importancia ambiental de cada impacto: 8 - 10 Importancia muy alta (MA), 6 - 8 Importancia alta (A), 4 - 6 Importancia media (M), 2 - 4 Importancia baja (B) y 0 - 2 Importancia muy baja (MB).

\section{RESULTADOS Y DISCUSIÓN}

Evaluación técnica del riego. En las parcelas experimentales evaluadas se observó el incremento de la presión a lo largo de los laterales de riego, debido a la disposición de los laterales a favor de la pendiente del terreno con ganancia de presión, siendo más notorio en la Argentina cuya pendiente es del $32 \%$. En la revisión detallada de los sistemas de riego se observaron básicamente fugas en las tuberías y obstrucción en los emisores de riego, que tienen consecuencia sobre la presión y el caudal de descarga de los emisores y afectan tanto la uniformidad como la distribución del frente de humedecimiento en el suelo (ver figura 4). 


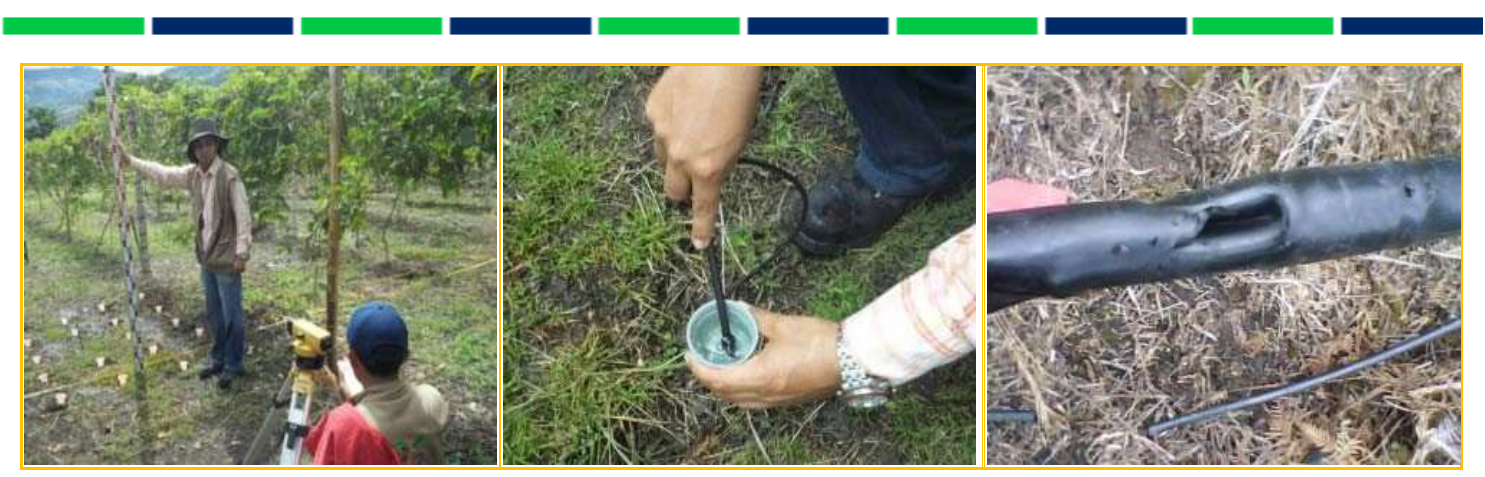

Figura 4. Medición de la pendiente del terreno, aforo del emisor y daños en la tubería

Es común observar que los microaspersores están expuestos a continuos daños por los operarios debidos su ubicación entre las calles, lo que ocasiona bajas en la uniformidad y en las eficiencias de riego. Por lo anterior, se recomienda ubicar los emisores en línea con las plantas e instruir a los operarios sobre la precaución y el mantenimiento del sistema antes y después de aplicar un riego.

El coeficiente de uniformidad para los distintos métodos de riego fue muy afectado por la pendiente pronunciada del terreno (32\%) y como consecuencia se da una importante ganancia de presión, siendo altamente sensible a cualquier daño en el sistema de riego.

Se tomó como representativa la parcela experimental de la Plata, donde se instaló el método de riego por surcos. La pendiente del terreno resultó de 5.6\%, siendo limitante para el riego por surcos. El caudal práctico con la pendiente dada fue de 0.3 lps, ajustado a un adecuado manejo según las condiciones del terreno. El método de riego por surcos resultó con una eficiencia alta (88.2\%), como consecuencia de la escasa longitud del surco (18 m) y las características franco - arcillosas del suelo. Se hallan los parámetros del riego para la instalación y posterior evaluación (ver tabla 2).

Tabla 2. Funciones y valores del riego por surcos

\begin{tabular}{|l|c|}
\hline Parámetro & Ecuación / valor \\
\hline Tiempo de avance (Ta) & $18 \mathrm{~min}$ \\
\hline $\begin{array}{l}\text { Función de infiltración acumulada } \\
\left(\mathrm{I}_{\text {cum }}\right)\end{array}$ & $1.53 \mathrm{t}$ \\
\hline Infiltración básica $\left(\mathrm{I}_{\mathrm{b}}\right)$ & $0.55 \mathrm{~cm} / \mathrm{hr}$ \\
\hline Longitud $(\mathrm{L})$ & $18 \mathrm{~m}$ \\
\hline Pendiente $(\mathrm{S})$ & $5.62 \%$ \\
\hline Función de avance $(\mathrm{X})$ & $4.35 \mathrm{t}(\mathrm{min})^{0.524}$ \\
\hline Lámina neta $\left(\mathrm{d}_{\mathrm{n}}\right)$ & $52 \mathrm{~mm}$ \\
\hline $\begin{array}{l}\text { Tiempo de aplicación }(\mathrm{Tap})=\mathrm{T}_{\mathrm{a}}+ \\
\text { T } 2\end{array}$ & $58.3 \mathrm{~min}$ \\
\hline Lámina bruta $\left(\mathrm{d}_{\mathrm{b}}\right)$ & $5.9 \mathrm{~cm}$ \\
\hline Percolación & $5.7 \%$ \\
\hline Escorrentía & $6.1 \%$ \\
\hline Eficiencia de aplicación - CU & $88.2 \%-83.8 \%$ \\
\hline
\end{tabular}


Se realizó un diseño experimental en bloques completos al azar para las variables Coeficiente de Uniformidad y Uniformidad de
Distribución teniendo cinco (5) métodos de riego (tratamientos) y el factor de bloqueo: caudal o presión del sistema (ver tabla 3).

Tabla 3. Coeficientes de uniformidad para caudal y presión en los métodos de riego evaluados.

\begin{tabular}{|c|l|l|l|l|l|}
\hline $\begin{array}{c}\text { Método de } \\
\text { riego }\end{array}$ & $\begin{array}{c}\text { Parcela } \\
\text { Experimental }\end{array}$ & $\begin{array}{c}\text { CUq } \\
\mathbf{( \% )}\end{array}$ & $\begin{array}{c}\text { CUp } \\
\mathbf{( \% )}\end{array}$ & $\begin{array}{c}\text { UDq } \\
\mathbf{( \% )}\end{array}$ & $\begin{array}{c}\text { UDp } \\
\text { (\%) }\end{array}$ \\
\hline \multirow{2}{*}{ Goteo } & La Plata & 99.3 & 99.7 & 82.5 & 89.6 \\
\cline { 2 - 6 } & La Argentina & 75.0 & 62.9 & 66.1 & 49.6 \\
\hline \multirow{2}{*}{ Microaspersión } & La Plata & 95.5 & 89.4 & 95.1 & 87.9 \\
\cline { 2 - 6 } & La Argentina & 92.3 & 97.4 & 90.7 & 94.1 \\
\hline Surcos & La Plata & 83.8 & NA & 80.9 & NA \\
\hline Microtubo & La Argentina & 61.2 & 57.1 & 58.2 & 56.1 \\
\hline \multirow{2}{*}{ Tradicional } & La Plata & 38.2 & NA & 35.1 & NA \\
\cline { 2 - 6 } & La Argentina & 30.5 & NA & 28.5 & NA \\
\hline
\end{tabular}

Leyenda: CUq y CUp coeficiente de uniformidad para caudal y presión respectivamente, UDq y UDp Uniformidad de distribución para caudal y presión respectivamente NA no aplica para surcos por riego no presurizado, NA no aplica para microtubo y tradicional por la compleja variabilidad de presiones en el sistema siendo no sostenible.1

La hipótesis nula planteada para los tratamientos fue: no existe diferencia significativa a un nivel del $5 \%$ entre los promedios de los coeficientes de uniformidad y uniformidad de distribución evaluados para los distintos métodos de riego aplicados en pasifloras.

La hipótesis nula planteada para los bloques fue: No existe diferencia significativa entre promedios de bloques (parámetro de uniformidad evaluado) en el riego de pasifloras. La pruebas de hipótesis se soporta mediante el análisis de varianza, el cual determina diferencias significativas entre los tratamientos de riego, ya que $\mathrm{Fc}>\mathrm{Ft} 0.05$ (4.88).

Aplicando el comparador Tukey (? = 0.05 ), cuyo valor es 21.5 , resulta que los métodos de riego por goteo, riego por microaspersión y riego surcos no presentan diferencias significativas entre sí, pero si con relación a los métodos de riego por microtubo y riego tradicional. Se observa, que el método de surcos aplicado en las condiciones del presente experimento, en suelo franco arcilloso, longitud inferior a $20 \mathrm{~m}$, descarga baja (0.3 lps) y dosificada en la cabecera con válvula de control y tiempo para infiltrar la lámina neta, determinan coeficientes de uniformidad de riego altos.

Los coeficientes de uniformidad y la uniformidad de distribución evaluados no presentan diferencias significativas en los propósitos de la evaluación tendientes a calificar la eficiencia de los métodos de riego. Después de realizar los ajustes del sistema, los coeficientes de uniformidad se calificaron excelente para goteo y microaspersión, aceptable para surcos e inaceptable para el riego por los métodos de microtubo y tradicional (ver tabla 1 ).

Los rendimientos promedios anuales de granadilla y maracuyá durante el periodo de investigación (enero 2010 a mayo 2012) fueron de 15.2 y de 32.5 ton/ha respectivamente sin diferencias significativas ( $9=0.05)$ para los distintos tratamientos bajo riego, debido a la constante del fenómeno climático de la Niña (fenómeno ENOS) que imperó en el suroccidente del departamento 
del Huila, donde estaban ubicadas las parcelas experimentales con valores de lluvia superiores de aproximadamente 2.5 veces sobre el promedio histórico.

Evaluación Socio-económica. El diagnóstico permitió determinar que el núcleo familiar lo conforman 4 personas en promedio, con área promedio de 1.3 hectáreas por productor, constituyéndose asociaciones de pequeños agricultores. Se deduce que la producción de pasifloras no es exclusiva de los hombres, además no se lleva un registro organizado de los costos de producción, ni especificación sobre las inversiones y ganancias.

Según las encuestas, los productores de los municipios anotados no están expuestos a periodos prolongados de déficit hídrico, por tal razón la implementación de riego es considerada una actividad secundaria que demanda inversión importante, siendo el sistema de riego tradicional poco eficiente. El agua para los sistemas de riego es captada en las quebradas, nacimientos y acueductos veredales para ser conducida hasta tanques de almacenamiento. Los métodos de riego más empleado son el microtubo y el método tradicional, seguido de la microaspersión. Los propios productores asumen la labor del riego y en pocas ocasiones contratan mano de obra para esta labor.

La inversión promedio por hectárea según las encuestas fue de aproximadamente siete millones novecientos cincuenta mil pesos (\$7'950.000), con inversión promedia en riego de seiscientos setenta y cinco mil pesos (\$675.000), que corresponde al 9.6\% de la inversión total. Los productores indican que no cuentan con un sistema de medida del agua para determinar el volumen total empleado.

Llontop 1999, considera que al norte del Perú, el cultivo ha permitido a las familias rurales afrontar los costos de producción y adquirir infraestructura para otras actividades como la caficultura, constituyéndose en el principal componente del mejoramiento del nivel de vida.

Evaluación ambiental del riego. La evaluación ambiental complementa la evaluación técnica y permite valorar con mayor equidad la eficiencia general de los sistemas de riego, para la selección del método más eficiente desde el punto de vista técnico, socio-económico y ambiental con miras a la sustentabilidad de la producción agrícola. Con la evaluación ambiental se recopiló información sobre el riego, los impactos ambientales asociados al riego y las medidas para enfrentar el cambio climático.

El $80 \%$ de los encuestados tiene conocimiento sobre el cambio climático y su incidencia, mencionando algunas de las siguientes estrategias para su mitigación: no construir más represas hidroeléctricas, implementar sistemas de riego, conocer la dinámica del clima para el manejo del cultivo, efectuar modelaciones para prever sucesos climáticos, construir almacenamientos de agua, implementar campañas sobre reforestación, uso razonable de los recursos y la aplicación de las energías alternativas.

Los impactos positivos tienen una mayor frecuencia y son inferiores a los impactos negativos, resaltando el aumento de la producción, el suministro de agua en forma oportuna y adecuada, la aplicación de abonos y la producción de fruta permanente. Como medidas para fortalecer los beneficios de un sistema de riego están: implementar un sistema de riego ajustado a las condiciones del cultivo, riego oportuno, mantenimiento continuo, fertirriego, manejo fitosanitario, aplicar agricultura de precisión, conocer las variables climáticas, dosificar el riego $\mathrm{y}$ planear todas las actividades agrícolas (ver tabla 4). 
Tabla 4. Impactos ambientales asociados al riego por el método de la encuesta

\begin{tabular}{|c|c|c|}
\hline Impacto Ambiental & $\begin{array}{c}\text { Número de } \\
\text { encuestados }\end{array}$ & Frecuencia \\
\hline \multicolumn{3}{|c|}{ IMPACTOS POSITIVOS } \\
\hline Balance hídrico edáfico & 3 & 15 \\
\hline Facilita la aplicación de abonos & 6 & 30 \\
\hline Disponibilidad del recurso hídrico & 3 & 15 \\
\hline $\begin{array}{l}\text { Suministro de agua en forma oportuna y } \\
\text { adecuada }\end{array}$ & 8 & 40 \\
\hline Mayor vigor en las plantas & 4 & 20 \\
\hline Aumento en los ingresos & 3 & 15 \\
\hline Aumento en la producción & 13 & 65 \\
\hline Producción de fruta permanente & 5 & 25 \\
\hline Mayor calidad del producto & 4 & 20 \\
\hline \multicolumn{3}{|c|}{ IMPACTOS NEGATIVOS } \\
\hline $\begin{array}{l}\text { Propagación de hongos y plagas en el } \\
\text { suelo }\end{array}$ & 7 & 35 \\
\hline Erosión & 3 & 15 \\
\hline Salinización & 4 & 20 \\
\hline Compactación & 2 & 10 \\
\hline Aumento del $\mathrm{pH}$ & 2 & 10 \\
\hline Encharcamiento & 3 & 15 \\
\hline $\begin{array}{l}\text { Contaminación de fuentes hídricas por } \\
\text { químicos }\end{array}$ & 2 & 10 \\
\hline Proliferación de malezas & 2 & 10 \\
\hline Conflictos por uso del agua & 2 & 10 \\
\hline Desperdicio del agua & 3 & 15 \\
\hline Mano de obra en mantenimiento & 2 & 10 \\
\hline
\end{tabular}

La baja frecuencia de los impactos negativos indica que son de poca presencia o magnitud y que los encuestados las perciben en menor grado, los principales problemas derivados de los sistemas de riego son plagas $\mathrm{y}$ enfermedades por hongos asociadas a exceso de humedad, desperdicio de agua, encharcamientos, erosión y pérdidas de las cosechas. La encuesta permite clasificar las afectaciones dependiendo del recurso, es decir suelo, agua, ambiente o social (ver tabla $5)$. 
Impactos en el proceso productivo de las pasifloras y uso eficiente del agua de riego

Tabla 5. Impactos ambientales asociados al riego organizados

\begin{tabular}{|c|c|c|}
\hline COMPONENTE & \multicolumn{2}{|c|}{ POSITIVO } \\
\hline \multirow{7}{*}{ SUELO } & Balance hídrico edáfico & $\begin{array}{l}\text { Arrastre de materia orgánica y lavado de } \\
\text { Nutrientes }\end{array}$ \\
\hline & Facilita la aplicación de abonos & $\begin{array}{l}\text { Propagación de hongos y plagas en el } \\
\text { suelo }\end{array}$ \\
\hline & & Erosión \\
\hline & & Salinización \\
\hline & & Lixiviación \\
\hline & & Compactación \\
\hline & & Encharcamiento \\
\hline \multirow{3}{*}{ AGUA } & $\begin{array}{l}\text { Disponibilidad de agua en el } \\
\text { predio }\end{array}$ & $\begin{array}{l}\text { Aumenta el drenaje superficial en el } \\
\text { predio }\end{array}$ \\
\hline & $\begin{array}{l}\text { Aplicación del riego en forma } \\
\text { oportuna y adecuada }\end{array}$ & $\begin{array}{l}\text { Contaminación de fuentes hídricas por } \\
\text { Químicos }\end{array}$ \\
\hline & & Disminución del caudal en fuentes hídricas \\
\hline \multirow[t]{3}{*}{ AGUA } & & Acumulación de sales \\
\hline & & Eutrofización \\
\hline & & Desperdicio de agua \\
\hline \multirow{8}{*}{ AMBIENTE } & Mejora el microclima en el cultivo & Modifica los ecosistemas \\
\hline & Mayor vigor de las plantas & Aumenta la temperatura \\
\hline & Mayor calidad del fruto & Contaminación por químicos \\
\hline & & Deterioro paisajístico \\
\hline & & Proliferación de malezas \\
\hline & & $\begin{array}{l}\text { Proliferación de plagas y enfermedades al } \\
\text { Cultivo }\end{array}$ \\
\hline & & Caída de flores \\
\hline & & Perdida de plantas \\
\hline \multirow{6}{*}{ SOCIAL } & Aumento en los ingresos & Costos de instalación \\
\hline & Valorización de la tierra & Robo de partes o accesorios del sistema \\
\hline & Generación de empleo & Daño del sistema por labores culturales \\
\hline & Aumento en la producción & Conflictos por uso del agua \\
\hline & Producción de fruta permanente & Desplazamiento de la mano de obra \\
\hline & & Mano de obra para el mantenimiento \\
\hline
\end{tabular}


Para la mitigación de las afectaciones negativas derivadas del riego, recomiendan hacer un control periódico tanto del riego como del estado fitosanitario del cultivo, evaluar la viabilidad del sistema de riego dependiendo de las condiciones del clima y la disponibilidad hídrica, hacer estimativo de la cantidad de agua aplicada, control de la escorrentía, capacitación en riego, implementar la unidad de filtrado y construir sistemas de drenaje. Se debe tener precaución en las labores culturales, no deteriorar los sistemas instalados y controlar además la contaminación por residuos sólidos $\mathrm{y}$ atmosféricos derivados de la aplicación de fertilizantes y químicos.

Las labores complementarias que benefician el cultivo son: la aplicación de abonos, fumigación de plagas, la limpieza alrededor del tallo denominada plateo, la aplicación de microorganismos al suelo y la fertilización. Es de vital importancia conocer los impactos ambientales del riego, para concientizar sobre la necesidad de seleccionar un método de riego adecuado, hacer el mantenimiento periódico y la evaluación técnica que permita conocer el estado de funcionamiento del sistema de riego, así como identificar $\mathrm{y}$ tomar las medidas para cuantificar las afectaciones y tomar los correctivos adecuados en el área de cultivo.

Al respecto Llontop 1999, afirma que los cultivos de granadilla y maracuyá que se desarrollan en un envidiable agroecosistema, con gran potencial biótico (flora, fauna y policultivo) y abiótico (suelos, materia orgánica y agua disponible) permiten cumplir con los propósitos gubernamentales de competitividad, equidad social $\mathrm{y}$ sostenibilidad ambiental. Por esto se debe controlar el riego para potencializar la competitividad de los productores.

El mejor escenario resultó al riego por goteo (E2), seguido de riego por microaspersión (E1) y por último surcos (E3). En total se analizaron 31 impactos de los cuales 12 fueron positivos y 19 negativos (tabla 6). Esta calificación permite determinar que todos los impactos no son evidentes, pero con la ampliación del área irrigada podrían ser importantes. Además de identificar y priorizar los impactos, es necesario idear estrategias para cuantificarlos y poner en práctica las medidas sugeridasEstimar el consumo de agua en cada uno de los tres híbridos de Palma Aceitera (Elaeis guineensis Jacq) utilizando el método de Balance de Masas.

Tabla 6. Orden de viabilidad de escenarios (método de riego) según la calificación ambiental

\begin{tabular}{|c|c|c|c|c|c|c|c|c|c|}
\hline $\begin{array}{l}\text { Método de } \\
\text { riego }\end{array}$ & $\begin{array}{l}\text { Importancia } \\
\text { Impacto }\end{array}$ & MA & $\mathbf{A}$ & $\mathbf{M}$ & B & MB & $\begin{array}{c}\text { Orden de } \\
\text { importancia } \\
\text { Positivo }\end{array}$ & $\begin{array}{c}\text { Orden de } \\
\text { importancia } \\
\text { Negativo }\end{array}$ & $\begin{array}{l}\text { Orden de } \\
\text { viabilidad }\end{array}$ \\
\hline \multirow{2}{*}{$\begin{array}{c}\text { Microaspersión } \\
\left(E_{1}\right)\end{array}$} & + & 0 & 1 & 1 & 5 & 5 & \multirow{2}{*}{1} & \multirow{2}{*}{2} & \multirow{2}{*}{2} \\
\hline & - & 0 & 0 & 0 & 3 & 18 & & & \\
\hline \multirow{2}{*}{$\begin{array}{c}\text { Goteo } \\
\left(E_{2}\right)\end{array}$} & + & 0 & 0 & 3 & 6 & 3 & \multirow{2}{*}{2} & \multirow{2}{*}{3} & \multirow{2}{*}{1} \\
\hline & - & 0 & 0 & 0 & 2 & 19 & & & \\
\hline \multirow{2}{*}{$\begin{array}{c}\text { Surcos } \\
\left(E_{3}\right)\end{array}$} & + & 0 & 0 & 0 & 2 & 10 & \multirow{2}{*}{3} & \multirow{2}{*}{1} & \multirow{2}{*}{3} \\
\hline & - & 0 & 0 & 1 & 6 & 14 & & & \\
\hline
\end{tabular}

Muy Alta (MA): 8 - 10; Alta (A): 6 - 8; Medio (M): 4 - 6; Bajo (B): 2 -4; Muy Bajo (MB): 0 - 2 
El 87.5\% de los encuestados consideraron que el método de microaspersión es el de mayor consumo de energía y así mismo es el más costoso, éste método junto con el riego por surcos son los que pueden generar más empleo 56,3\%. En cuanto al método de riego por goteo, consideran que aunque es costoso por su mantenimiento, es el de mayor rendimiento en pasifloras. El riego por goteo es considerado como el mejor método porque es eficiente y económico, requiere menos agua, es puntual a la raíz, no aumenta la humedad relativa, no genera pérdidas mayores por viento, algunos agregaron que no necesita mucho mantenimiento, y es el más utilizado junto con aspersión.

\section{CONCLUSIONES}

Los coeficientes de uniformidad de riego en pasifloras por los métodos de goteo (99.3\%), microaspersión (95.5\%) y surcos (83.8\%) no presentan diferencias significativas entre sí, pero sí con relación a los métodos de riego por microtubo (61.2\%) y riego tradicional $(38.2 \%)$.

La eficiencia de riego evaluada a través del coeficiente de uniformidad califica los métodos de riego por goteo y microaspersión como excelentes, aceptable para surcos e inaceptable para los métodos de microtubo y tradicional.

Los rendimientos promedios anuales de granadilla y maracuyá fueron de 15.2 y de 32.5 ton/ha respectivamente sin diferencias significativas ( $=0.05$ ) para los distintos tratamientos bajo riego, debido a la influencia del fenómeno de la Niña (fenómeno ENOS) que imperó en las áreas experimentales.

Los impactos positivos más destacados son: la disponibilidad de agua, la calidad de la fruta y la producción permanente; los impactos negativos están asociados al uso deficiente del riego resaltándose el mal drenaje, la presencia de plagas y enfermedades, la erosión y la pérdida de las cosechas.
Las medidas para fortalecer los beneficios de un sistema de riego son: implementar sistemas de riego ajustados a las condiciones del cultivo, riego oportuno, mantenimiento continuo, fertirriego, manejo fitosanitario, implementar la agricultura de precisión, prever los cambios climáticos, dosificar el riego, control de la escorrentía, capacitación en riego, implementar unidades de filtrado, construir sistemas de drenaje y planear las actividades agrícolas.

\section{Agradecimiento}

A Colciencias y a la Vicerrectoría de Investigación y Proyección Social de la Universidad Surcolombiana por proveer los recursos para el financiamiento de la investigación, y al apoyo logístico de la Corporación Centro para la gestión tecnológica de pasifloras del departamento del Huila - CEPASS.

REFERENCIAS

Arboleda, J. (1994). Una propuesta para la identificación y evaluación de impactos ambientales. Crónica Forestal y del Medio Ambiente 9: 71-81.

Cadena Productiva Frutícola. (2006). Manual técnico del cultivo de maracuyá (Passiflora edulis L). Departamento del Huila. Secretaría Técnica. Neiva. 34 p.

Corpoica (2002). Corporación Colombiana en Investigación Agropecuaria - Universidad Pontificia Bolivariana, Centro de Desarrollo Tecnológico de Frutales CDTF. IV Seminario Nacional Frutales de clima frio moderado. Medellín, Noviembre 20 al 22 del 2002

Christiansen J., E. (1942). Irrigation by Sprinkling. Bulletin 670. University of California. Agricultural Experimental Station Berkeley, California. 124 p

Gurovich, L. (1993). Validación experimental de un modelo de simulación para el diseño, operación y evaluación del riego por surco. Ciencia e Investigación Agraria, Volumen 20 № 1, 1993. ISSN 0304- 5609, Pontificia Universidad Católica de Chile. pág. 33-46 
Llontop, J.A. (1999). El cultivo de la granadilla en la región norte del Perú. Centro de Investigación, Asesoría y Promoción (CICAP). 19 - 28 p

Keller, J.; Karmeli, D. (1975). Trickle irrigation design. Rain Bird Sprinkler Manufacturing Corporation, $133 \mathrm{p}$

MADR, (2006). Ministerio de Agricultura y Desarrollo Rural, Gobernación del Huila, Fondo Nacional de Fomento Hortifrutícola - FNFH, Asociación Hortofrutícola de Colombia - Asohofrucol, Sociedad de Agricultores y Ganaderos del Valle del Cauca - SAG .Plan Frutícola, desarrollo de la fruticultura en el Huila. Neiva. $68 \mathrm{p}$

Torrente, A. (2011). Potencial de agua en el suelo y su relación hídrica en los cultivos de pasifloras, Granadilla (Passiflora ligularis Juss.) y Maracuyá (Passiflora edulis) en el Huila. Grupo de investigación GHIDA, Universidad Surcolombiana. Neiva -Huila, 133 pEstimar el coeficiente de cultivo (Kc) de los tres híbridos Tenera de Palma Aceitera (Elaeis guineensis Jacq) para la zona de La Concordia. 